\title{
Degree Theory beyond Continuous Maps
}

\author{
Louis Nirenberg \\ Courant Institute, New York University \\ 251 Mercer Street \\ New York, NY 10012 \\ e-mail: nirenl@cims.nyu.edu
}

I am very honoured and pleased to participate in the commemoration of the $50^{\text {th }}$ anniversary of the founding of the CWI of the Dutch Foundation Mathematical Centre.

The subject of my talk is concerned not with differential equations but with something that is used all the time in various nonlinear analytic problems, namely degree theory. It seems particularly appropriate to speak on this subject here since degree theory was developed by L.E.J. Brouwer. The infinite dimensional extension of degree theory, the Leray-Schauder degree, is a basic tool in attacking nonlinear differential equations. This talk describes some joint work with H. BREZIS [5][6] and is concerned with finite dimensional degree.

I recall the notion of degree (and its properties) of a map $u$ from one smooth $n$-dimensional compact oriented manifold $X$ without boundary to a connected one $Y$ of the same dimension. The degree measures, in a suitable sense, the number of times $Y$ is covered. It may be defined using homology theory but we describe it in more analytic terms:

Suppose $u$ is in $C^{1}$ and that $y \in Y$ is a regular value of the map, i.e., the preimage of $y, u^{-1}(y)$, consists of a finite number of points, $x_{1}, \ldots, x_{k}$ in $X$, and the Jacobian matrix $J_{u}$, in terms of local coordinates near $x_{j}$ and near $y$, is nonsingular at each $x_{j}$. If we choose local coordinates compatible with the given orientations on $X$ and $Y$, then degree of $u$ at $y$, denoted $\operatorname{deg}(u, X, y)$, counts the number of points $x_{j}$ in $u^{-1}(y)$ algebraically:

$$
\operatorname{deg}(u, X, y)=\sum_{i=1}^{k} \operatorname{sgn} \operatorname{det} J_{u}\left(x_{i}\right) .
$$

This number turns out to be independent of $y$, and is defined as the degree of the map $u$ from $X$ to $Y, \operatorname{deg}(u, X, Y)=\operatorname{deg} u$.

In case we put Riemannian metrics on $X$ and $Y$, then degree may be expressed by an integral, 
(1) $\operatorname{deg}(u, X, Y)=\frac{1}{\operatorname{Vol} Y} \int_{X} \operatorname{det} J_{u}(x) d \operatorname{Vol}(x)$.

Here $J_{u}$ is computed using geodesic normal coordinates about $x$, and geodesic normal coordinates about $u(x)$.

Here is another well known formula in case $X=\partial \Omega$, where $\Omega$ is a smooth bounded domain (open connected set) in $\mathbb{R}^{n}$, and $Y=S^{n-1}$. Suppose $u \in$ $C^{1}(X, Y)$. If $\widetilde{u}$ is any $C^{1}$ extension of $u$ inside $\Omega$, as a map into $\mathbb{R}^{n}$, then

(2) $\operatorname{deg}\left(u, \partial \Omega, S^{n-1}\right)=\frac{1}{|B|} \int_{\Omega} \operatorname{det} J_{\widetilde{u}} d x^{1} \cdots d x^{n}$.

Here $|B|$ is the volume of the unit ball $B$ in $\mathbb{R}^{n}$.

The notion of degree extends to continuous maps from $X$ to $Y$, via approximation in $C(X, Y)$, by $C^{1}$ maps from $X$ to $Y$. This is because of the following fundamental fact.

There exists $\delta_{0}>0$ depending only on $X$ and $Y$ such that if $u, v \in C^{1}(X, Y)$ and the distance from $u$ to $v$ measured in the $C^{0}$ norm (this depends on metrics on $X$ and $Y$ ), dist $_{C^{0}}(u, v)$, is less than $\delta_{0}$, then

$$
\operatorname{deg} u:=\operatorname{deg}(u, X, Y)=\operatorname{deg}(v, X, Y)=: \operatorname{deg} v .
$$

Here are some further properties of degree.

1. Degree is invariant under homotopy, i.e. if we deform a map $u \in C(X, Y)$, depending continuously on a parameter, then the degree does not change.

2. If $\operatorname{deg} u \neq 0$ then $Y \subset u(X)$.

Degree is also defined for a map from $X$ to $Y$ in case $X$ has a boundary: Suppose $X$ is an open set with compact closure in an open oriented $n$-manifold $\widetilde{X}$, and that $Y$ is an open connected oriented $n$-manifold. Then for $u \in C(\bar{X}, Y)$, if $y \in Y \backslash u(\partial X)$, the degree $\operatorname{deg}(u, X, y)$ is defined. It is also invariant under continuous deformation $u_{t}$ of the map $u$ and continuous deformation $y_{t}$ of $y$, depending on a continuous parameter $t$, provided

$$
y_{t} \notin u_{t}(\partial X) \quad \forall t .
$$

One more simple fact about degree: Suppose $X$ is the unit ball $B$ in $\mathbb{R}^{n}$ and that $u$ maps $\bar{B}$ into $\mathbb{R}^{n}$. If $y \in \mathbb{R}^{n} \backslash u(\partial B)$ then

(4) $\operatorname{deg}(u, B, y)=\operatorname{deg}\left(\frac{u-y}{|u-y|}, S^{n-1}, S^{n-1}\right)$.

In recent years, in work on some variational problems connected with some nonlinear partial differential equations, it has become of interest to extend degree to some classes of maps which need not be continuous. In 1983 BREZIS 
\& Coron [4] studied maps $u$ from $S^{2}$ to $S^{2}$ belonging to the Sobolev space $W^{1,2}\left(S^{2}, S^{2}\right)$, i.e., having square integrable first derivatives. Such maps just miss being continuous, if the derivatives use $L^{p}$ integrable, for $p>2$, the maps would be continuous, by the Sobolev embedding theorem. It seems natural to extend degree theory to such maps via the expression on the right hand side of (1), for if $u \in W^{1,2}\left(S^{2}, S^{2}\right)$ then $\operatorname{det} J_{u}$ is in $L^{1}$. More generally, the integral in (1) makes sense for a map $u \in W^{1, n}\left(X^{n}, Y^{n}\right)$. Here we think of $Y$ as smoothly embedded in some $\mathbb{R}^{N}$, and that $u(x) \in Y$ for almost every $x$ in $X$.

QUESTION. Is the corresponding expression on the right hand side of (1) an integer? If so, does it have the usual properties of degree?

As above, functions $u$ in $W^{1, p}, p>n$ are continuous, but they need not be in case $p=n$. It is rather clear that the answer to the question is yes if smooth maps from $X$ to $Y$ are dense in $W^{1, n}(X, Y)$. At this point let me make some side remarks in connection with density of smooth maps.

Suppose $M$ and $N$ are compact manifolds of possibly different dimensions; $M$ may have boundary, but $N$ not. $N$ is assumed to be smoothly embedded in $\mathbb{R}^{s}$ for some $s$. Under what conditions is $C^{1}(M, N)$ dense in $W^{1, p}(M, N)$ ? The answer is

a) Yes if $p>m=\operatorname{dim} M$; this is quite easy since such maps are continuous, by Sobolev embedding.

b) Yes is $p=m$. The proof uses Poincaré's inequality, and is essentially due to Schoen \& Uhlenbeck [10].

c) For $p<m$ there is the following result of Bethuel [2]: The answer is

$$
\text { yes } \Longleftrightarrow \quad \Pi_{[p]}(N)=0 \text {. }
$$

Returning to degree theory for maps in Sobolev classes between manifolds, L. Boutet de Monvel and O. Gabber (see ref. [6] in [5]) considered maps $u$ in $W^{1,1 / 2}\left(S^{1}, S^{1}\right)$. In terms of the Fourier series expansion of $u$,

$$
u(\theta)=\sum a_{j} e^{i j \theta}
$$

with $|u(\theta)| \equiv 1$ a.e. $\theta$, the $W^{1,1 / 2}$ norm of $u$ is given by

$$
\|u\|_{W^{1,1 / 2}}^{2}=\sum|j|\left|a_{j}\right|^{2} .
$$

If $u$ were smooth then $\operatorname{deg} u$ is just the winding number of the image about $S^{1}$, which is given by

$$
\begin{aligned}
\operatorname{deg} u & =\frac{1}{2 \pi i} \oint_{S^{1}} \frac{d u}{u}=\frac{1}{2 \pi i} \oint \bar{u} d u \\
& =\sum j\left|a_{j}\right|^{2}
\end{aligned}
$$


by Parseval. Thus for $u \in W^{1,1 / 2}$, the series (5) is absolutely convergent, and Boutet de Monvel and Gabber show that it is an integer. It is not obvious that for, say, a continuous map $u$ from $S^{1}$ to $S^{1}$, the series (5) in terms of the Fourier coefficients of $u$, is summable and equals an integer.

More generally, for $B$ the unit ball in $\mathbb{R}^{n}$, consider a map $\widetilde{u} \in W^{1, n}\left(B, \mathbb{R}^{n}\right)$. The trace $u$ of $\widetilde{u}$ on $S^{n-1}$ is well defined as a function in $W^{1-1 / n, n}\left(S^{n-1}, \mathbb{R}^{n}\right)$. Suppose $|u(x)|=1$ a.e. Look at formula (2). The right hand side makes sense. Does the left hand side? We cannot use formula (1) for the left hand side, since $u=$ trace $\widetilde{u}$, has just fractional derivatives; the right hand side of (1) has no sense.

Boutet de Monvel and Gabber pointed out that for maps from $S^{1}$ to $S^{1}$, an appropriate class of maps for which to define degree, and which includes maps in $W^{1,1 / 2}$, and continuous maps, is the class $V M O$. What Brezis and I have done is to carry this out for $V M O$ maps from general $X$ to $Y$.

Let me first recall the class $B M O$, functions of bounded mean oscillation. (It was introduced by F. John in connection with work in elasticity theory.) Let $X$ be an $n$ dimensional Riemannian manifold without boundary and with some injectivity radius $r_{0}$. The class of real functions $f$ in $B M O(X)$ is a slight enlargement of $L^{\infty}(X)$.

BMO: An integrable real function $f$ is in $B M O$ if

$$
\|f\|_{B M O}=\sup _{B \subset X} f_{B}\left|f-f_{B} f\right|<\infty .
$$

Here $B$ represents a geodesic ball in $X$ with radius $<r_{0}, f_{B}$ denotes taking the average over $B$.

Modulo adding constants, $B M O$ is a Banach space, with the norm given in (6). An equivalent norm is

(7) $\|f\|_{*}=\sup _{B \subset X}\left\{f_{B}|f(y)-f(z)|\right.$

This second expression enables one to define $B M O$ maps from $X$ into any metric space.

A typical example of a $B M O$ function in $\mathbb{R}^{n}$ is

$$
f=\log \left|x-x_{0}\right| \text {. }
$$

Now the space of continuous functions is not dense in $B M O$. D. SARASON [9] studied the closure of the space of continuous functions in $B M O$, calling it $V M O$ because, as he proved, it is characterized by the following property: 
(8) $f$ in $B M O$ belongs to $V M O \Longleftrightarrow \lim _{|B| \rightarrow 0} \oint_{B}\left|f-\oint_{B} f\right|=0$

Here $|B|$ denotes the volume of $B$.

EXAmples. In $\mathbb{R}^{n}$, the function $\log |x|$ is not in $V M O$, but $|\log | x||^{\alpha}, 0<$ $\alpha<1$ is; so also is $\log |\log | x||$. For $X$ a compact manifold as before, without boundary, $W^{1, n}(X) \subset V M O(X)$. This is proved with the aid of Poincaré's inequality. More generally, the Sobolev space $W^{s, p}(X)$ with derivatives of order $s, s$ real, with $0<s<n$, and $s p=n$, is in $V M O$. A good recent reference for $B M O$ and $V M O$ is STEIN [11]. A map $u: X \rightarrow \mathbb{R}^{n}$ is in $B M O$ (or $V M O$ ) if each component is.

Degree. Consider, as before, our compact, oriented, manifolds $X, Y$ of dimension $n$, without boundary. We suppose that they have Riemannian metrics and that $Y$ is smoothly embedded in $\mathbb{R}^{N}$ for some $N$. Let $u$ be a $V M O$ map from $X$ to $Y, u \in V M O(X, Y)$, meaning, $u \in V M O\left(X, \mathbb{R}^{N}\right)$ and $u(x) \in Y$ for almost every $x \in X$.

We extend the notion of degree via approximation, in the BMO topology, by continuous maps. This is done in [5] as follows. For $u$ in $\operatorname{VMO}\left(X, \mathbb{R}^{n}\right)$, $0<\varepsilon<r_{0}$, the function

$$
\bar{u}_{\varepsilon}(x)=f_{B_{\varepsilon}(x)} u,
$$

(here $B_{\varepsilon}(x)$ is the geodesic ball of radius $\varepsilon$ centred at $x$ ) is continuous and tends to $u(x)$ in the $B M O$ topology as $\varepsilon \rightarrow 0$. It does not take values in $Y$; however, for $\varepsilon$ small, it lies close to $Y$, namely, we have:

$$
\operatorname{dist}\left(\bar{u}_{\varepsilon}(x), Y\right) \leq \oint_{B_{\varepsilon}(x)}\left|u-\bar{u}_{\varepsilon}(x)\right| \longrightarrow 0 \quad \text { as } \quad \varepsilon \rightarrow 0,
$$

by Sarason's characterization (8). Now define

$$
u_{\varepsilon}(x)=P \bar{u}_{\varepsilon}(x)
$$

where $P$ is projection to closest point on $Y$.

Definition $\operatorname{deg}(u, X, Y)=\operatorname{deg}\left(u_{\varepsilon}, X, Y\right)$ for $\varepsilon$ small.

Since, for $\varepsilon$ small, the maps $u_{\varepsilon}$ are all homotopic, it follows that the maps all have the same degree. A principal result is:

Theorem 1. For $u \in V M O(X, Y), \exists \delta>0$ such that for every $v \in V M O(X, Y)$, with

$$
\begin{aligned}
\|u-v\|_{B M O} & \leq \delta \\
\operatorname{deg}(v, X, Y) & =\operatorname{deg}(u, X, Y) .
\end{aligned}
$$


A natural question is whether this result holds with a fixed $\delta$ independent of $u$, i.e. does the analogue of (3) hold? The answer is no.

\section{SOME PROPERTIES:}

i) By Theorem 1, the degree is invariant under continuous homotopy in $V M O(X, Y)$. It does not depend on the Riemannian metrics nor the particular embedding of $Y$ in $\mathbb{R}^{N}$.

ii) If $\operatorname{deg}(u, X, Y) \neq 0$ then

(9) $\operatorname{Ess} R(u)=Y$.

To explain, note that since $u$ is defined a.e., the range of $u$ is not well defined.

Definition Ess $R(u)$ is the smallest closed set $\sum$ in $Y$ such that

$$
u(x) \in \sum \quad \text { for almost all } \quad x \in X
$$

It exists.

(9) asserts that there is no open ball $B$ in $Y$ with $u(x) \in Y \backslash B$ a.e.

REMARK. The image of $u$ may cover $Y$ infinitely many times. Example: $X=Y=S^{1}$, and

$$
\begin{aligned}
u & =e^{i f(\theta)}, \\
f(\theta) & =\theta+\zeta(\theta)|\log | \theta-\pi|| ;
\end{aligned}
$$

here $\zeta$ is a smooth cutoff (bump) function with support near $\theta=\pi$.

Further properties of degree are given in [5]. The proofs are rather straightforward. A useful fact there is the following:

Remark. If $u \in B M O\left(X, \mathbb{R}^{N}\right)$ and $F$ is a uniformly continuous map of $\mathbb{R}^{N}$ to $\mathbb{R}^{T}$ then

$$
F \circ u \in B M O\left(X, \mathbb{R}^{T}\right) .
$$

However, in its dependence on $u$, as a map from $B M O\left(X, \mathbb{R}^{N}\right)$ to $B M O\left(X, \mathbb{R}^{T}\right)$, it need not be continuous. (This is true even if $F$ is Lipschitz.) It is continuous at every $u$ in $V M O\left(X, \mathbb{R}^{N}\right)$.

$V M O$ is not the most general class for which degree, having the usual properties, may be defined. $\operatorname{deg}(u, X, Y)$ may also be defined for $u \in B M O(X, Y)$ provided $u$ is close to $V M O$ in the $B M O$ topology, i.e. that $M_{0}(u)$ defined below, is small. 
Sarason [9] showed that $\exists A>0$ such that

$$
M_{0}(u) \leq \operatorname{dist}_{B M O}\left(u, \operatorname{VMO}\left(X, \mathbb{R}^{N}\right)\right) \leq A M_{0}(u),
$$

where for $a \leq r_{0}$,

$$
M_{a}(u)=\sup _{\substack{x \in X \\ \varepsilon \leq a}} \oint_{B_{\varepsilon}(x)}\left|u-\bar{u}_{\varepsilon}(x)\right|
$$

and

$$
M_{0}(u)=\lim _{a \searrow 0} M_{a}(u)
$$

FurTher REMARKS.

a) (1) holds for $u \in W^{1, n}(X, Y)$.

b) (2) holds if $u \in W^{1-1 / n, n}\left(\partial \Omega, S^{n-1}\right)$ and $\widetilde{u} \in W^{1, n}\left(\Omega, \mathbb{R}^{n}\right)$.

c) (4) holds if $u \in W^{1, n}\left(B, \mathbb{R}^{n}\right)$ and $|u(x)-y| \geq \delta>0$ for almost all $x \in S^{n-1}$.

Recently A. AbBondandalo [1] proved that $\operatorname{VMO}(X, Y)$ is homotopically equivalent to $C(X, Y)$ via the inclusion map. Also L. Greco, T. Iwaniec, C. Sbordone \& B. Stroffolini [7] defined degree for a class of Sobolev maps weaker than $W^{1, n}(X, Y)$, and which are not in $V M O$.

In [6] we take up the case that $X$ has compact closure in a larger manifold $\widetilde{X}$. For simplicity here, suppose $X$ is a bounded open set in $\mathbb{R}^{n}$, and that $Y=\mathbb{R}^{n}$. First one should explain what is meant by $B M O(\Omega)$. There are apparently various possibilities:

$$
\|f\|_{B M O}=\sup _{\bar{B} \subset \Omega} f_{B}\left|f-\oint_{B} f\right|<\infty,
$$

in fact one may use any norm, not just the Euclidean norm. Another possibility is to consider balls $B$ "well inside $\Omega$," i.e. for some $\tau$ fixed, $0<\tau<1$,

$$
\|f\|_{B M O, \tau}=\sup _{\substack{B r(x) \\ r \leq \tau \operatorname{dist}(x, \partial \Omega)}} \oint_{B_{r}(x)}\left|f-\oint_{B_{r}(x)} f\right|
$$

P. Jones [8] has shown that these norms are all equivalent, and that, furthermore, (private communication) if $V M O(\Omega)$ is the completion of $C(\bar{\Omega})$ in the $B M O$ topology, then $C_{0}^{\infty}(\Omega)$ is dense in $V M O(\Omega)$. See [6] for modifications of Jones' arguments.

Now, for some $y$ in $\mathbb{R}^{n}$ we wish to define $\operatorname{deg}(u, \Omega, y)$ in case $u \in V M O\left(\Omega, \mathbb{R}^{n}\right)$.

For continuous maps $u$ one assumes that

(10)y $\notin u(\partial \Omega)$. 
However, a map $u \in V M O$ need not have a trace on $\partial \Omega$. We replace assumption (10) by

$$
\text { (11) }\left\{\begin{array}{l}
\exists \delta, r_{0}>0 \quad \text { such that } \\
\int_{B_{r}(x)}^{f}|u(z)-y| \geq \delta \quad \forall x \in \Omega, \quad \text { with } \quad r \equiv \frac{1}{2} \text { dist }(x, \partial \Omega) \leq r_{0}
\end{array}\right.
$$

Then $\operatorname{deg}(u, \Omega, y)$ can be well defined, and usual properties hold.

Under some circumstances one would like to have property (4). We derive this property for a particular class of maps $u$ in $\operatorname{VMO}\left(\Omega, \mathbb{R}^{n}\right)$ for which we can define a trace on $\partial \Omega$ if $\partial \Omega$ is smooth.

In [6] we also answer a question raised by H. Amann. Suppose $X$ and $Y$ are, as above, compact without boundaries and $u$ and $v$ are in $V M O(X, Y)$ - so the degrees of $u$ and $v$ are defined. Suppose $u$ and $v$ are connected by a homotopy $H$ belonging not to $C([0,1], V M O(X, Y))$, but to $V M O(X \times[0,1] ; Y)$. Is it true that $\operatorname{deg} u=\operatorname{deg} v$ ? Under suitable conditions on $H$ for t near 0 and 1 , Cor. 3 in [6] asserts that the answer is yes. For further results see [6].

BREzIS [3] is an excellent expository article on our results.

ACKNOWLEDGMENT. The work was partly supported by grants ARO-DAAL03-92-G-0143 and NSF-DMS-94-00912. The author also wishes to thank the Forschungsinstitut of ETH.

\section{REFERENCES}

1. A. Abbondandalo. On the homotopy type of $V M O$, to appear in Top'l methods in Nonlinear Analysis.

2. F. Bethuel (1991). The approximation problem for Sobolev maps between two manifolds. Acta Math. 167, 153-206.

3. H. Brezis (1995). Degree Theory: old and new, to appear in Proc. Frascati Conf.

4. H. Brezis, J. M. Coron (1983). Large solutions for harmonic maps in two dimensions. Comm. Math. Phys. 92, 203-215.

5. H. Brezis, L. Nirenberg (1995). Degree theory and BMO; Part I: Compact manifolds without boundaries. Selecta Math., New Ser. 1, 197-263.

6. H. Brezis, L. Nirenberg Degree theory and BMO; Part II: Compact manifolds with boundaries. Submitted to Selecta Math.

7. L. Greco, T. Iwaniec, C. Sbordone, B. Stroffolini. Degree formulas for maps with nonintegrable Jacobian (to appear).

8. P. Jones (1980). Extension theorems for BMO, Math. J. 29, 41-66.

9. D. Sarason (1975). Functions of vanishing mean oscillation. Trans. Amer. Math. Soc. 207, 391-405.

10. R. Schoen and K. Uhlenbeck (1983). Boundary regularity and the Dirichlet problem for harmonic maps. J. Diff. Geom. 18, 253-268.

11. E. Stein (1993). Harmonic Analysis. Princeton University Press. 